\title{
Nab-Paclitaxel and Gemcitabine in Advanced Pancreatic Cancer: The One-year Experience of the National Cancer Institute of Naples
}

\author{
ALESSANDRO OTTAIANO ${ }^{1}$, MONICA CAPOZZI ${ }^{1,6}$, CHIARA DE DIVITIIS $^{1,6}$, CLAUDIA VON ARX $^{1}$, \\ ELENA DI GIROLAMO ${ }^{2}$, GUGLIELMO NASTI ${ }^{1}$, ERNESTA CAVALCANTI ${ }^{3}$, \\ FABIANA TATANGELO ${ }^{4}$, GIOVANNI ROMANO ${ }^{5}$, ANTONIO AVALLONE ${ }^{1}$ and SALVATORE TAFUTO ${ }^{1,6}$ \\ Departments of ${ }^{1}$ Abdominal Oncology and ${ }^{5}$ Abdominal Surgery, Istituto Nazionale \\ Tumori di Napoli “G. Pascale” IRCCS, National Cancer Institute, Naples, Italy; \\ Units of ${ }^{2}$ Endoscopy, ${ }^{3}$ Laboratory Unit and ${ }^{4}$ Pathology Unit of the Department of Diagnostic Pathology and Laboratory, \\ Istituto Nazionale Tumori di Napoli "G. Pascale" IRCCS, National Cancer Institute, Naples, Italy; \\ ${ }^{6}$ ENETS Center of Excellence, Multidisciplinary Group for Neuroendocrine Tumors in Naples, Naples, Italy
}

\begin{abstract}
Background: Pancreas adenocarcinoma is the sixth cause of cancer-related death worldwide with an increasing mortality in the Western countries. Recently, the association between nab-paclitaxel (nab-P) and gemcitabine (GEM) has significantly improved progression-free and overall survival. Patients and Methods: Patients affected by metastatic pancreas adenocarcinoma were treated at the Department of Abdominal Oncology of the National Cancer Institute of Naples from July 2015 to July 2016 with nab-P at $125 \mathrm{mg}$ per square meter of body-surface area followed by GEM at 1,000 $\mathrm{mg}$ per square meter on days 1,8 and 15 every 4 weeks. Computed tomography (CT) was performed every three months of therapy. Toxicity was graded with National Cancer InstituteCommon Toxicity Criteria (NCI-CTC) v4.0. Objective responses were evaluated with Response Evaluation Criteria in Solid Tumors (RECIST). Analysis of time-to-progression is only descriptive. Pain was evaluated with a visual analogue scale (VAS). Results: Twenty-three patients were treated. Median age was 67 years (range $=45-81$ ); 8 patients were $\geq 70$ years old. Performance status (PS) Eastern Cooperative Oncology Group (ECOG) was 2 in 8 patients, 1 in 10 and 0 in 5. Twelve patients presented with diffuse hepatic metastases, 4 with carcinosis, 7 with more than one organ involvement. Nab$P$ was reduced at $100 \mathrm{mg}$ per square meter in all patients. The
\end{abstract}

Correspondence to: Dr. Alessandro Ottaiano, Department of Abdominal Oncology, Istituto Nazionale Tumori di Napoli "G. Pascale" IRCCS, National Cancer Institute, via Mariano Semmola, 80131, Naples, Italy. Tel/Fax: +39 0815903510, e-mail: ale.otto@libero.it

Key Words: Nab-Paclitaxel, gemcitabine, pancreatic cancer, chemotherapy, clinical study. most common G3/G4 adverse events were neutropenia $(13.0 \%$ G4, 8.6\% G3; none was febrile), neuropathy (30.4\% G3) and asthenia (G3 17.3\%). The disease control rate was $43.4 \%$ (partial response + stable disease $(P R+S D)$ 10/23). The median time-to-progression was 7.9 months $(95 \%$ confidence interval $(C I)=5.8-11.2)$. After three months of therapy the PS improved in 14 patients, as well as pain in 18 patients. Conclusion: We present an experience with nab-P and GEM association in a series with poor PS and highly metastatic disease relatively to a previous randomized study. The schedule is feasible, with nab-P at $100 \mathrm{mg}$ per square meter achieving a good disease control rate, as well as a clinical benefit.

Advanced pancreas adenocarcinoma (APC) is the fourth cause of cancer-related death worldwide showing an increasing mortality rate in the Western countries. Most pancreatic cancers $(\sim 95 \%)$ are inoperable at diagnosis for locally advanced or metastatic disease. Unfortunately, the prognosis of APC is dismal with a median survival of about six months (1).

Gemcitabine (GEM) is the mainstay of systemic treatment of APC. Other drugs have been used for the treatment of APC, including cisplatin, fluoropirimidines, irinotecan, taxanes, pemetrexed; both single agents or doublets failed to achieve encouraging improvements versus GEM monotherapy (2). New targeted therapies also failed to ameliorate the prognosis of APC patients (3). However, two studies showed a significant advantage in survival: the study by Moore et al. (4) involving patients treated with GEM plus erlotinib who obtained a clinically irrelevant median survival increase of two weeks and the study by Conroy et al. (5) involving patients treated with fluororuracil, leucovorin, irinotecan and oxalipltain (FOLFIRINOX) who obtained a median increase in survival of 4.3 months accompanied, 
however, by a significant increase in some G3/G4 toxicities (febrile neutropenia, thrombocytopenia, fatigue, vomiting, diarrhea, neuropathy). Furthermore, in the last study, patients were excluded from treatment if they had a Performance status (PS) Eastern Cooperative Oncology Group (ECOG) $\geq 2$, altered bilirubin levels, any significant history of cardiac disease and being older than 75 years. These conditions (one or more) are often present in the real clinical practice.

Recently, Von Hoff et al. (6) showed that the association of GEM with nab-paclitaxel (nab-P) in APC determined a +1.8month median survival gain (median survival of 6.7 months for GEM vs. 8.5 for GEM plus nab-P; Hazard ratio $(\mathrm{HR})=0.72$; $95 \%$ confidence interval $(\mathrm{CI})=0.62-0.83 ; p<0.001)(3)$. Toxicity profile was manageable; in particular, the proportion of patients with $\mathrm{G} 3 / \mathrm{G} 4$ toxicities was similar in the two treatment arms (43\% with GEM vs. 50\% with nab-P plus GEM). This study enrolled patients with Karnofsky PS $\geq 70 \%$ (which is equivalent to ECOG 0-1) and normal bilirubin levels; however, significant history of cardiac disease or age were not exclusion criteria.

In the present study, we report a real-practice one-year experience of the Department of Abdominal Oncology of the National Cancer Institute of Naples from July 2015 to July 2016 with nab-P and GEM in APC treatment.

\section{Patients and Methods}

Patients affected by metastatic pancreas adenocarcinoma were treated at the Department of Abdominal Oncology of the National Cancer Institute of Naples from July 2015 to July 2016. The criteria for patients' treatment were: life expectancy of at least three months, PS ECOG $<3$, adequate renal, liver and cardiac functions. Any other medical conditions, excluding chemotherapy, were discussed in a multidisciplinary context in order to evaluate alternative strategies. The treatment consisted on nab-P at $125 \mathrm{mg}$ per square meter of body-surface area followed by GEM at 1,000 mg per square meter on days 1,8 and 15 every 4 weeks. All patients, who had histologically or cytologically confirmed advanced APC, had never previously received chemotherapy for metastatic disease. Patients included in this study could not undergo radiotherapy or other locoregional treatments. The chemotherapy was continued until progression of disease or unacceptable toxicity. Computed tomography (CT) was performed every three months of therapy. Toxicity was graded with National Cancer Institute-Common Toxicity Criteria (NCI-CTC) v4.0. Objective responses were evaluated with Response Evaluation Criteria in Solid Tumors (RECIST). Pain was evaluated with a visual analogue scale (VAS) from 0 (no pain) to 10 (maximum pain), filled by the patients. VAS results are only descriptive. Considering the characteristics of this cohort and the expected time-to-progression (TTP) of the disease, PS and pain were evaluated before and after 3 months therapy, to avoid interference of progressive disease (PD). In patients who stopped therapy early, PS and pain were evaluated at withdrawn from nab-P/GEM treatment. TTP was defined as the time elapsed from treatment start to progression of the cancer as it occurred first. Analysis of TTP is descriptive; data for survival are still immature. No " $a$ priori" study design was required; the end-point of this study was
Table I. Characteristics of patients and treatment exposure.

\begin{tabular}{lc}
\hline Characteristics & No. \\
\hline Age, years & 67 \\
Median & $45-81$ \\
Range & \\
Gender & 13 \\
Male & 10 \\
Female & \\
Performance status & 5 \\
0 & 10 \\
1 & 8 \\
2 & \\
Site of primary tumor & 7 \\
Head & 9 \\
Body & 7 \\
Tail & \\
Site of metastases & 12 \\
Liver & 4 \\
Peritoneum & 7 \\
More than one site & \\
Nab-P/GEM administrations & 13 \\
Median & $3-32$ \\
Range &
\end{tabular}

Nab-P, Nab-paclitaxel; GEM, gemcitabine.

Table II. Summary of G3/G4 adverse events.

\begin{tabular}{lrrrrrr}
\hline Toxicity & \multicolumn{2}{c}{ G3 } & & \multicolumn{2}{c}{ G4 } \\
\cline { 2 - 3 } \cline { 6 - 7 } & No & $\%$ & & No & $\%$ \\
\hline Neuropathy & 7 & 30.4 & & 0 & 0.0 \\
Neutropenia & 2 & 8.6 & & 3 & 13.0 \\
Asthenia & 4 & 17.3 & & 0 & 0.0 \\
Hyperbilirubinemia & 3 & 13.0 & & 0 & 0.0 \\
Alkaline phosphatase & 3 & 13.0 & & 0 & 0.0 \\
Hyperglycemia & 2 & 8.6 & & 0 & 0.0 \\
Thrombocytopenia & 2 & 8.6 & & 0 & 0.0 \\
\hline
\end{tabular}

to describe activity and efficacy of nab-P/GEM in a real practice consecutive cohort of APC patients treated at our Institution. All patients signed an informed consent for treatment.

\section{Results}

Patients' characteristics. Twenty-three patients affected by metastatic adenocarcinoma of the pancreas were treated from July 2015 to July 2016. Patients' characteristics are listed in Table I. Median age was 67 years (range $=45-81$ ); 8 patients were $\geq 70$ years old. Performance status (PS) ECOG was 2 in 8 patients, 1 in 10 and 0 in 5 . Twelve patients presented with diffuse hepatic metastases, 4 with carcinosis, 7 with more than one organ involvement. 


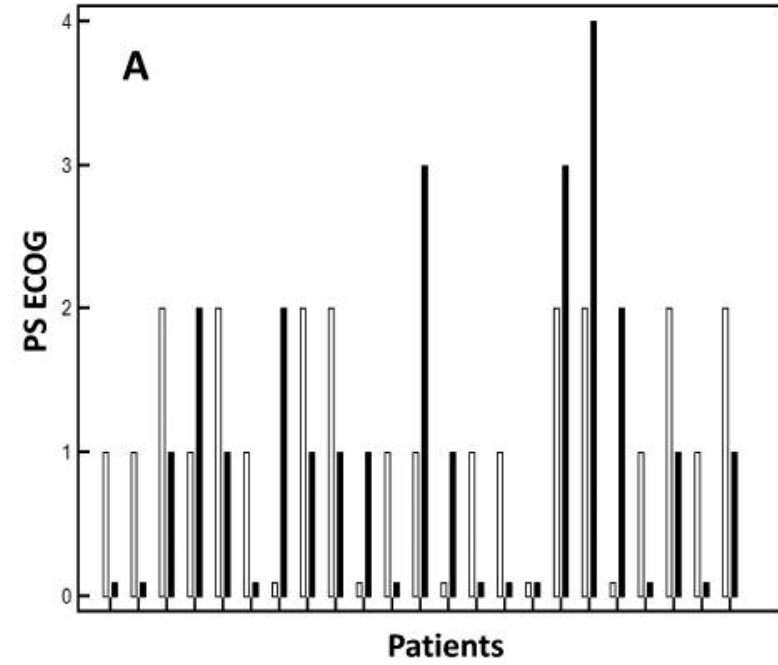

Before therapy

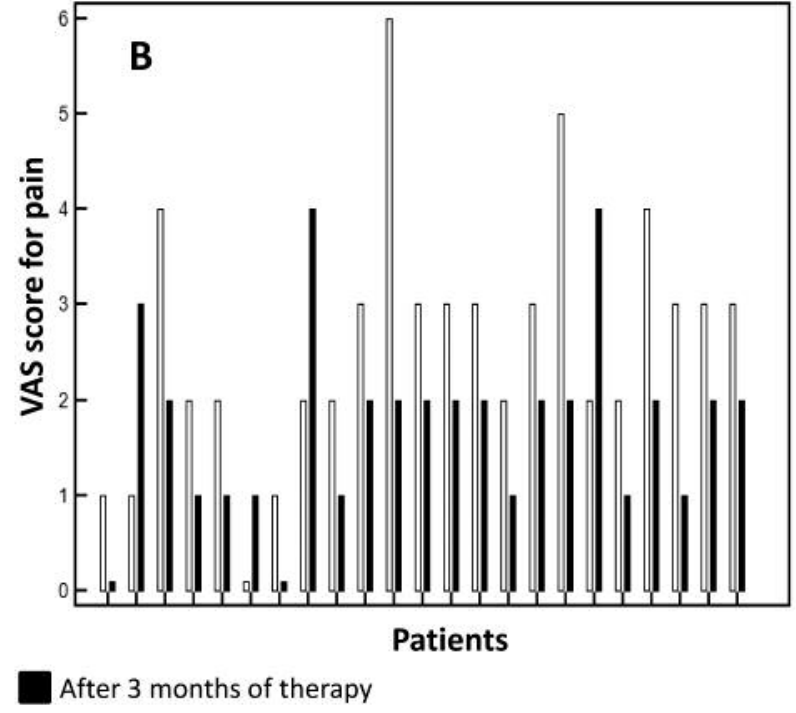

After 3 months of therapy

Figure 1. Bar plot of performance status Eastern cooperative oncology group (PS ECOG) (A) and visual analogue scale (VAS) for pain results (B) for all patients before and after three months of treatment with nab-paclitaxel (nab-P) and gemcitabine (GEM).

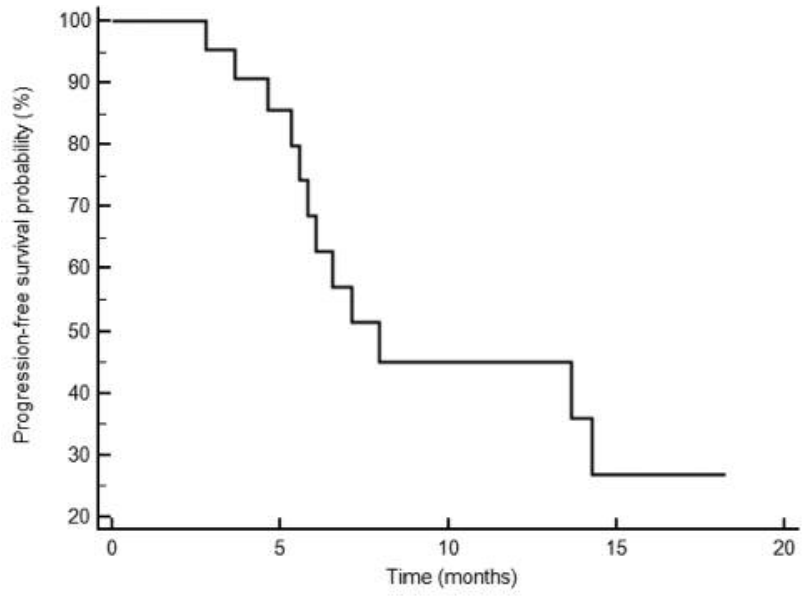

Figure 2. Time-to-progression Kaplan-Meier curve.

Treatment exposure. Patients older than 70 years started nab$\mathrm{P}$ treatment at $100 \mathrm{mg} / \mathrm{square}$ meter $(\mathrm{sm})$. Considering ages (14 patients $>65$ years old) and poor PS of our cohort, Nab$\mathrm{P}$ was reduced at $100 \mathrm{mg} / \mathrm{sm}$ at the first occurrence of asthenia and/or neutropenia G2 (in 10 patients after the first dose of chemotherapy and in 5 patients at third dose). GEM was reduced in five patients at $800 \mathrm{mg} / \mathrm{sm}$. The patients received a median number of 13 administrations (range $=3$ 32 ) of therapy and the median treatment duration was 4.3 months (range $=0.4-10.9$ ).

Safety. The most frequent clinical toxicities G1/G2 included fatigue, nausea, anemia and neuropathy. Table II lists the
Table III. Efficacy estimates of nab-P/GEM.

\begin{tabular}{lc}
\hline & No. $(\%)$ \\
\hline Response to therapy & \\
Complete response & $0(0.0)$ \\
Partial response & $3(13.0)$ \\
Stable disease & $7(30.4)$ \\
Progressive disease & $13(56.6)$ \\
Median time-to-progression & 7.9 months $(95 \% \mathrm{CI}=5.8-11.2)$ \\
\hline
\end{tabular}

CI, Confidence interval.

treatment-related CTC G3/G4 adverse events. The most common G3/G4 adverse events were neutropenia (3 patients (pts), 13.0\% G4; 2 pts, $8.6 \%$ G3; none was febrile), neuropathy (7 pts, 30.4\% G3), asthenia (4 pts, G3 17.3\%). Thrombocytopenia occurred in 2 patients, $(8.6 \% \mathrm{G} 3)$. Hyperglycemia was the most frequently reported biochemical toxicity, resulting in clinical relevance only in two patients $(8.6 \%$ G3). Grade 3 levels of alkaline phosphatase and hyperbilirubinemia occurred in 3 patients $(13.0 \%)$. Treatment was interrupted for toxicity in 5 patients.

Antitumor activity, clinical benefit and time-to-progression. All patients were available for radiological response evaluation; eight patients discontinued treatment early (five for toxicity, three for progression) but were, nonetheless, available for the radiological response evaluation CT-based. The disease control rate was $43.4 \%$ (partial response + stable disease $(\mathrm{PR}+\mathrm{SD})$ 10/23); PR was registered in three patients (response 
rate $=13.0 \%$ ) (Table III). As measured by VAS, after three months of therapy, pain ameliorated in 18 patients, PS improved in 14 patients (Figure 1). At the time of the analysis of these data, 11 patients are still alive and 5 are pursuing a second-line treatment; twelve patients progressed and seven died. The median TTP was 7.9 months (95\% CI=5.8-11.2) (Figure 2).

\section{Discussion}

APC is an aggressive and chemotherapy-resistant neoplasm; many drugs failed to improve survival versus GEM single agent over the last fifteen years. Intensive research is attempting to improve quantity and quality of life of patients affected by metastatic pancreas adenocarcinoma. Very recently, the association of nab-P and GEM showed a +1.8 month median survival gain in a well-designed randomized clinical trial $(6,7)$. Here, we report a mono-institutional experience with nab-P and GEM association in a realpractice series of metastatic pancreas adenocarcinoma patients with poor PS and highly metastatic disease relatively to the previous randomized studies.

Data from randomized trials may suffer of low reliability due to the a priori selection of patients; in particular, the selection of younger and good PS patients may limit the extension of results in the clinical practice. With respect to these aspects, it is well-known that age and performance status are strong prognostic factors in hepatobiliary and pancreas neoplasms $(8,9)$. The studies by Moore et al. (4) and Conroy et al. (5) did not enroll the patients if they had a PS ECOG $\geq 2$ and/or altered bilirubin levels. Additionally, the study by Conroy et al. (5) excluded patients with any significant history of cardiac disease or older than 75 years. Thus, in this regard, study series from the real "unselected" clinical practice may help to confirm or support the results from clinical trials.

Considering the characteristics of our cohort (PS 0-2, highly metastatic disease, ages over 65 years) we decided to start or reduce nab-P at $100 \mathrm{mg} / \mathrm{sm}$ at first occurrence of G2 asthenia or neutropenia. The schedule was feasible and achieved a good disease control rate, as well as a clinical benefit; patients with PR or SD showed also a cancer antigen 19-9 reduction (data not shown). The safety profile was consistent with previous studies and G3 adverse events resolved without specific treatments.

It is known that paclitaxel bound to albumin crosses endothelial cells and reaches and concentrates in tumors by receptor-mediated transcytosis and enhanced permeation and retention effect (10). Additionally, it has been demonstrated that nab-P enhances GEM levels in tumors (11). Hypothetically, reduction of nab-P does not disrupt these positive effects and preserves the anti-neoplastic properties of GEM.

In summary, this strategy did not subtract efficacy to therapy and adherence to treatment was high; future research could help to clarify if nab-P at $100 \mathrm{mg} / \mathrm{sm}$ is "non-inferior" to a $125 \mathrm{mg} / \mathrm{sm}$ schedule, particularly in older ages.

\section{References}

1 Worni M, Guller U, White RR, Castleberry AW, Pietrobon R, Cerny T, Gloor B and Koeberle D: Modest improvement in overall survival for patients with metastatic pancreatic cancer: A trend analysis using the surveillance, epidemiology, and end results registry from 1988 to 2008. Pancreas 42: 1157-1163, 2013.

2. Kamisawa T, Wood LD, Itoi T and Takaori K: Pancreatic cancer. Lancet 388: 73-85, 2016.

3 Ciliberto D, Staropoli N, Chiellino S, Botta C, Tassone P and Tagliaferri P: Systematic review and meta-analysis on targeted therapy in advanced pancreatic cancer. Pancreatology 16: 249258, 2016.

4 Moore MJ, Goldstein D, Hamm J, Figer A, Hecht JR, Gallinger S, Au HJ, Murawa P, Walde D, Wolff RA, Campos D, Lim R, Ding K, Clark G, Voskoglou-Nomikos T, Ptasynski M and Parulekar W: Erlotinib plus gemcitabine compared with gemcitabine alone in patients with advanced pancreatic cancer: A phase III trial of the National Cancer Institute of Canada Clinical Trials Group. J Clin Oncol 25: 1960-1966, 2007.

5 Conroy T, Desseigne F, Ychou M, Bouché O, Guimbaud R, Bécouarn Y, Adenis A, Raoul JL, Gourgou-Bourgade S, de la Fouchardière C, Bennouna J, Bachet JB, Khemissa-Akouz F, PéréVergé D, Delbaldo C, Assenat E, Chauffert B, Michel P, MontotoGrillot $\mathrm{C}$ and Ducreux M: FOLFIRINOX versus gemcitabine for metastatic pancreatic cancer. N Engl J Med 364: 1817-1825, 2011.

6 Von Hoff DD, Ervin T, Arena FP, Chiorean EG, Infante J, Moore M, Seay T, Tjulandin SA, Ma WW, Saleh MN, Harris M, Reni M, Dowden S, Laheru D, Bahary N, Ramanathan RK, Tabernero J, Hidalgo M, Goldstein D, Van Cutsem E, Wei X, Iglesias J and Renschler MF: Increased survival in pancreatic cancer with nabpaclitaxel plus gemcitabine. N Engl J Med 36: 1691-1703, 2013.

7 Goldstein D, El-Maraghi RH, Hammel P, Heinemann V, Kunzmann V, Sastre J, Scheithauer W, Siena S, Tabernero J, Teixeira L, Tortora G, Van Laethem JL, Young R, Penenberg DN, Lu B, Romano A and Von Hoff DD: Nab-Paclitaxel plus gemcitabine for metastatic pancreatic cancer: Long-term survival from a phase III trial. J Natl Cancer Inst 107(2): pii: dju413, 2015. doi: 10.1093/jnci/dju413

8 Smith BJ and Mezhir JJ: An interactive Bayesian model for prediction of lymph node ratio and survival in pancreatic cancer patients. J Am Med Inform Assoc 21: e203-211, 2014.

9 Maréchal R, Demols A, Gay F, De Maertelaere V, Arvanitaki M, Hendlisz A and Van Laethem JL: Prognostic factors and prognostic index for chemonaïve and gemcitabine-refractory patients with advanced pancreatic cancer. Oncology 73: 41-51, 2007.

10 Danhier F, Danhier P, De Saedeleer CJ, Fruytier AC, Schleich N, des Rieux A, Sonveaux P, Gallez B and Préat V: Paclitaxelloaded micelles enhance transvascular permeability and retention of nanomedicines in tumors. Int J Pharm 20: 399-407, 2015.

11 Noh I, Kim HO, Choi J, Choi Y, Lee DK, Huh YM and Haam S: Co-delivery of paclitaxel and gemcitabine via CD44-targeting nanocarriers as a prodrug with synergistic antitumor activity against human biliary cancer. Biomaterials 53: 763-774, 2015.

Received January 25, 2017

Revised March 1, 2017

Accepted March 2, 2017 